\title{
Relação entre os níveis de atividade física e qualidade de vida de idosos sedentários e fisicamente ativos
}

\author{
Relationship between physical activity levels and quality of life of sedentary and physically active \\ elderly
}

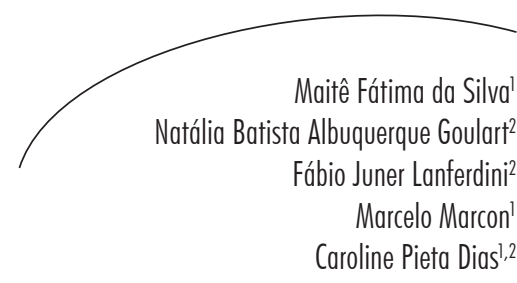

Resumo

Introdução: O nível de atividade física pode interferir na capacidade funcional, influenciando na saúde e qualidade de vida dos idosos. Objetivo: Comparar os níveis de atividade física e qualidade de vida entre idosos que praticam exercícios físicos regulares e idosos sedentários, e verificar a associação entre o nível de atividade física e a qualidade de vida nos grupos. Métodos: Foram avaliados 50 idosos do sexo feminino $(\mathrm{n}=34)$ e masculino $(\mathrm{n}=16)$, com idade de 70,24 $\pm 8,8$ anos, divididos em dois grupos - sedentários $(G 1, n=25)$ e praticantes de exercícios físicos regulares $(G 2, n=25)$. Realizou-se entrevista individual, aplicando-se Anamnese; Questionário Internacional de Atividade Física (IPAQ) e Perfil de Saúde de Nottingham (PSN). A normalidade dos dados foi testada por meio do teste de Shapiro-Wilk, utilizando-se ainda os testes U de Mann-Whitney (qualidade de vida) e Qui-Quadrado (nível de atividade física). A associação entre o nível de atividade física e a qualidade de vida foi avaliada por meio do teste $\operatorname{ETA}^{2}(\mathrm{p} \leq 0,05)$. Resultados: Em relação ao PSN, o G1 apresentou reduzida qualidade de vida, comparado ao G2. Já para o IPAQ, somente o G1 apresentou diferença significativa ( $p=0,00)$ em relação ao $G 2$. Além disso, para o $G 1,50 \%$ do nível de atividade física foram associados à qualidade de vida, enquanto o G2 apresentou 64\%. Conclusões: De acordo com os resultados encontrados no presente estudo, idosos praticantes de exercícios físicos e idosos sedentários apresentam bom nível de atividade física. Entretanto, idosos praticantes de exercícios regulares possuem maior nível de atividade física, explicando a melhor qualidade de vida neste grupo.

\section{Abstract}

Introduction: The physical activity level may interfere on functional capacity, influencing on elderly health and quality of life. Objective: To compare physical activity levels and quality of life among elderly who take regular physical exercise and sedentary ones, and the association between these variables. Methods: The study analyzed 50 elderly

Curso de Educação Física. Faculdade da Serra Gaúcha. Caxias do Sul, RS, Brasil.

2 Laboratório de Pesquisa do Exercício, Escola de Educação Física. Universidade Federal do Rio Grande do Sul. Porto Alegre, RS, Brasil.

Palavras-chave: Idosos. Qualidade de vida. Atividade física. Sedentarismo. 
people (women, $\mathrm{n}=34$, male, $\mathrm{n}=16$ ) aged $70.24 \pm 8.8$ years, divided into two groups: sedentary $(\mathrm{G} 1, \mathrm{n}=25)$ and physically active $(\mathrm{G} 2, \mathrm{n}=25)$. Anamnesis, International Physical Activity Questionnaire (IPAQ) and the Nottingham Health Profile (NHP) were applied. Normality data was tested using the Shapiro-Wilk. Mann-Whitney U test (quality of life) and Chi-Square test (physical activity) were also used. The association between physical activity level and quality of life was assessed by ETA ${ }^{2}$ test $(\mathrm{p} \leq 0.05)$. Results: In relation to the NHP, the G1 had reduced quality of life when compared to G2. For IPAQ, only G1 showed a significant difference $(p=0.00)$ in comparison with G2. In addition, $50 \%$ of the physical activity level was associated with life quality on G1, while G2 showed 64\%. Conclusions: In this study, both physically active and sedentary elderly have good level of physical activity. However, regular physically active elderly showed a higher level of physical activity, which explains the better quality of life of this group.

\section{INTRODUÇÃO}

Nos últimos 50 anos, a população brasileira modificou seu perfil, revelando uma nova realidade. O aumento do percentual de idosos, somado à diminuição da natalidade, são situações atuais em um país considerado jovem, mas com características que nos preparam para uma população idosa. ${ }^{1}$

Segundo dados do Instituto Brasileiro de Geografia e Estatística (IBGE), ${ }^{2}$ em 2030, o número de idosos deverá superar o de crianças e adolescentes em cerca de quatro milhões, diferença que aumentará para 35,8 milhões em 2050. Com o aumento da expectativa de vida, segundo a Organização Mundial da Saúde (OMS), ${ }^{3}$ o Brasil será o sexto país do mundo com o maior número de pessoas idosas até 2025.

O envelhecimento pode ser conceituado de diferentes formas, dentre as quais a definição mais difundida é a perda da eficiência dos processos envolvidos na manutenção da homeostase do organismo, implicando decréscimo da viabilidade ou aumento da vulnerabilidade ao estresse. Dentro desse contexto, as modificações morfológicas e funcionais relacionadas ao envelhecimento constituem uma das maiores preocupações para os profissionais da área da saúde, sobretudo no que se refere à prevenção de doenças e melhora da qualidade de vida. ${ }^{4}$

Neste sentido, a atividade física regular e sistematizada ou exercícios físicos regulares têm
Key words: Elderly. Quality of life. Physical activity. Sedentary lifestyle. demonstrado serem capazes de minimizar os efeitos deletérios do envelhecimento, como a redução na massa muscular, colaborando para a manutenção da capacidade física e autonomia do idoso. ${ }^{5}$ Atualmente, já é possível observar um maior número de programas de exercícios físicos voltados aos idosos.

Entretanto, em virtude de a maioria da população não possuir acesso a programas de atividade física orientada, por questões financeiras ou ausência de interesse pessoal, torna-se importante manter os níveis de atividade física por meio das tarefas de vida diárias (caminhar, higiene pessoal, levantar sem auxílio), bem como atividades instrumentais da vida diária (utilizar meios de transporte, lavar sua roupa, fazer compras e administrar os próprios medicamentos). ${ }^{6}$ Sendo assim, manter as atividades de vida diária, como varrer a casa ou ir ao supermercado, também pode ser um recurso importante para minimizar os efeitos degenerativos provocados pelo envelhecimento, que são agravados com o sedentarismo.

Faz-se necessário compreender o perfil atual dos idosos, abrangendo aspectos de atividade física, bem como qualidade de vida, por meio de pesquisas referentes ao envelhecimento. Assim, o presente estudo tem como objetivos comparar os níveis de atividade física e qualidade de vida de idosos que praticam atividades físicas regulares com idosos considerados sedentários, bem como verificar se existe associação entre 
o nível de atividade física e a qualidade de vida em ambos os grupos.

\section{MÉTODOS}

Trata-se de estudo descritivo, correlacional de corte transversal. A seleção da amostra foi realizada por meio da amostragem nãoprobabilística por acessibilidade.

A amostra foi constituída por 50 idosos do sexo feminino $(\mathrm{n}=34)$ e masculino $(\mathrm{n}=16)$, com idade média de 70,24 $\pm 8,8$ anos divididos em dois grupos: sedentários $(\mathrm{n}=25)$ e praticantes de atividade física regular $(\mathrm{n}=25)$. Os idosos praticantes de atividade física regular foram recrutados em grupos de atividade para a terceira idade e academias, adotando-se como critério de inclusão a realização das atividades de vida diária, bem como a prática de atividades físicas orientadas e sistematizadas com frequência de no mínimo duas vezes por semana, com duração de pelo menos uma hora por sessão, realizadas em grupo ou individualmente. O grupo de idosos sedentários foi recrutado na comunidade em geral, sendo adotado como critério de inclusão a realização de atividades de vida diária e ausência da prática de atividades físicas regulares. Todos os participantes assinaram um termo de consentimento livre e esclarecido, e o estudo foi aprovado pelo Comitê de Ética e Pesquisa do Circulo Operário Caxiense, sob nº 0046.

Para a coleta de dados, foi utilizada uma anamnese composta por 34 questões, permitindo dessa maneira a caracterização da amostra. Foram obtidas informações socioeconômicas, de saúde e atividades.

Outro instrumento utilizado foi o IPAQ (International Physical Activity Questionnaire), que permite estimar o tempo semanal gasto na realização de atividades físicas de intensidade moderada a vigorosa e em diferentes contextos da vida (trabalho, tarefas domésticas, transporte e lazer). Há também a possibilidade de estimar o tempo despendido em atividades mais passivas (realizadas na posição sentada).
O questionário (forma longa, semana usual, versão 8) foi aplicado na forma de entrevista individual. Para utilização com pessoas idosas, nas questões do IPAQ foram incluídos exemplos de atividades que são comuns às pessoas desse grupo etário. Além disso, o campo para preenchimento das informações foi modificado, passando a ser requerido que o entrevistador registrasse o tempo médio habitual para cada dia da semana (segunda a domingo), ao invés de indicar apenas a frequência semanal e o tempo médio de realização dessas atividades físicas. ${ }^{8}$ Para a análise dos níveis de atividade física, foi usado o consenso entre o Centro de Estudos do Laboratório de Aptidão Física de São Caetano do Sul (CELAFISCS) e o Center for Disease Control, considerando os critérios de frequência e duração, que classifica as pessoas em muito ativa, ativa, irregularmente ativa e sedentária. ${ }^{9}$

O terceiro instrumento utilizado foi o Perfil de Saúde de Nottingham (PSN). Este questionário é um instrumento genérico de avaliação da qualidade de vida, e tem sido utilizado nos últimos anos para avaliação em idosos. O PSN fornece uma indicação breve e simples da percepção do indivíduo, relacionada ao seu bem-estar físico, social e emocional. Os 38 itens são baseados na classificação de incapacidade proposta pela OMS, e têm possibilidade de resposta "sim" ou "não". Os itens são divididos em seis domínios: habilidade física, nível energético, dor, reação emocional, qualidade do sono e interação social. A cada resposta "sim" é atribuído um ponto e quanto menor o escore, melhor a qualidade de vida. ${ }^{10}$

A normalidade dos dados foi testada por meio do teste de Shapiro-Wilk. A comparação entre os grupos para a variável qualidade de vida mensurada pelo Perfil de Saúde de Nottingham (PSN) foi avaliada por meio do teste $\mathrm{U}$ de Mann-Whitney. Para o nível de atividade física, mensurado pelo IPAQ, foi utilizado o teste do Qui-Quadrado. A verificação da associação entre o nível de atividade física e a qualidade de vida foi avaliada por meio do teste ETA ${ }^{2}$. O nível de significância adotado foi $\mathrm{p} \leq 0,05$. 


\section{RESULTADOS}

O teste U de Mann-Whitney demonstrou diferença entre os grupos em relação à variável qualidade de vida. O grupo 1 (G1) apresentou escore maior, representando qualidade de vida reduzida em relação ao grupo 2 (G2), como demonstrado na tabela 1.

Tabela 1 - Média e desvio padrão dos escores obtidos no PSN. Caxias do Sul, RS.

\begin{tabular}{lcc}
\hline \multicolumn{1}{c}{ Perfil de Nottingham (PSN) } & Grupo 1 & Grupo 2 \\
\hline Média & 10.64 & 5.24 \\
DP & 7.79 & 5.54 \\
\hline
\end{tabular}

G1: Grupo sedentário; G2: Grupo praticante de atividade física.

O teste do Qui-quadrado mostrou que o G1 apresentou diferença significativa $(\mathrm{p}=0,01)$ entre o nível de atividade física, enquanto o G2 não apresentou diferença significativa $(\mathrm{p}=0,054)$. As frequências são apresentadas na tabela 2.

Tabela 2. Frequência absoluta e relativa da classificação do nível de atividade física. Caxias do Sul, RS.

\begin{tabular}{lcccc}
\hline \multicolumn{1}{c}{ Nível de Atividade Física } & \multicolumn{2}{c}{$\mathrm{G} 1$} & $\mathrm{G} 2$ \\
\hline Frequência & $\mathrm{N}$ & $\%$ & $\mathrm{~N}$ & $\%$ \\
Muito ativo & 1 & 4 & 6 & 24 \\
Ativo & 15 & 60 & 14 & 56 \\
Irregularmente ativo & 6 & 24 & 5 & 20 \\
Sedentário & 3 & 12 & 0 & 0 \\
Total & 25 & 100 & 25 & 100 \\
\hline
\end{tabular}

G1: Grupo sedentário; G2: Grupo praticante de atividade física.

Em relação à associação entre as variáveis qualidade de vida e nível de atividade física pelo teste $\mathrm{ETA}^{2}$, verificou-se que, para o grupo sedentário, $50 \%$ do nível de atividade física estão associados à qualidade de vida. Entretanto, no grupo praticante de atividade física esta associação foi de $64 \%$.

\section{DISCUSSÃO}

De acordo com os dados apresentados na tabela 1, o G1 obteve escore maior no PSN quando comparado ao G2. Esses resultados mostram que idosos praticantes de exercícios físicos possuem melhor qualidade de vida. Pauli et al. ${ }^{11}$ afirmam que existe grande diferença de aptidão funcional entre o grupo de idosas que restringem seus níveis de atividade física às tarefas de vida diária e aquelas que acrescentam a participação regular em um programa supervisionado de atividades físicas variadas. Este comportamento diferenciado da aptidão funcional nos grupos de idosas pode interferir diretamente na qualidade de vida. Em outro estudo, Toscano \& Oliveira $^{12}$ observaram que mulheres idosas ativas apresentaram melhores resultados em aspectos relacionados à qualidade de vida, como capacidade funcional e estado geral de saúde. 
Na variedade de domínios que a qualidade de vida apresenta, existe um elemento básico e aliado a todos os outros, que é a capacidade para realizar movimentos corporais de forma eficiente. ${ }^{13}$ As dificuldades na locomoção, no manuseio de instrumentos ou na manutenção e adaptação de posturas nas diferentes tarefas do cotidiano demonstram a diminuição da autonomia do indivíduo idoso, determinando mudanças em sua qualidade de vida. Além disso, manter um estilo de vida ativo, segundo Ghorayeb \& Barros ${ }^{14}$ faz com que pessoas engajadas em algum tipo de prática de exercícios físicos apresentem redução dos riscos de surgimento ou progressão de doenças crônicas degenerativas.

Conforme apresentado na tabela 2 , os idosos classificados como ativos no IPAQ compõem a maioria em ambos os grupos, e esse percentual equivale a $60 \%$ no G1 e a $56 \%$ no G2. Podese analisar que o percentual de ativos do G2 é menor, o que pode ser explicado pelo fato de os idosos buscarem os exercícios físicos como forma de organizar uma rotina, e também por não desempenharem outras tarefas além das de ordem doméstica. Já no G1, pode-se afirmar que os idosos ativos, por mais que não façam exercícios físicos, ocupam seus dias com a rotina do lar, trabalho e atenção à família.

Um aspecto de destaque no desempenho de idosos ativos é sua importância para a execução das atividades da vida diária (AVDs), em particular aquelas que possibilitam uma vida independente. De acordo com Okuma, ${ }^{15}$ na medida em que um idoso sobe alturas de mais de $50 \mathrm{~cm}$, o mesmo é capaz de subir num ônibus com segurança, mantendo as interações que acontecem fora de casa, condição essencial para seu bem-estar. Além disso, é importante acrescentar que o American College of Sports Medicine (ACSM) ${ }^{16}$ afirma que, atualmente, quanto mais ativa é uma pessoa, menos limitações físicas ela tem.

Analisando a classificação "muito ativo" do IPAQ, observamos que no G1 apenas 4\% dos indivíduos são classificados nesse nível. Já no G2, 24\% são considerados muito ativos. Esses dados sugerem que os idosos praticantes de exercícios físicos apresentam maior disposição e possuem melhor desempenho na execução de suas tarefas, o que proporciona maior incentivo para a procura de mais atividades, tornandoos assim muito ativos. O mesmo pode ser observado em um estudo em que Mazo et al. ${ }^{17}$ relacionaram o domínio físico com o nível de atividade física e constataram que idosas mais ativas tinham energia suficiente para o dia a dia, boa capacidade de locomoção, satisfação com seu sono e com sua capacidade para exercer as atividades de vida diária e de trabalho.

Cosme et al. ${ }^{18}$ afirmam que o treinamento sistemático e específico das capacidades físicamotoras e funcionais contribui para a manutenção de níveis ótimos de aptidão física. Idosos submetidos a esse tipo de estímulo por no mínimo dois anos apresentam um perfil de condição física e funcional semelhante a de um adulto de meia idade. Portanto, a prática da atividade física colabora para uma velhice saudável, diminuindo ou retardando as perdas inerentes ao processo do envelhecimento. Além disso, a atividade física contribui de maneira fundamental para a manutenção da independência, e esta constitui um dos mais importantes fatores da qualidade de vida do idoso. ${ }^{19} \mathrm{~A}$ prática de qualquer atividade física é importante para um envelhecimento saudável e independente. Contudo, quando esta atividade é praticada como exercício físico, os melhores benefícios para a capacidade física são alcançados. ${ }^{20,21}$

O presente estudo mostrou que o G1 apresentou $12 \%$ dos participantes classificados como sedentários, pois estes praticam menos de dez minutos contínuos de alguma atividade física por semana. Já o G2 não apresentou nenhum idoso sedentário, confirmando, de acordo com o IPAQ, que todos praticam acima de 30 minutos de atividades físicas por dia. A prevalência da inatividade física foi observada por Hallal et al. ${ }^{22}$ em um estudo que revelou que $43,2 \%$ das mulheres entrevistadas, com idade de 60 a 69 anos $(\mathrm{n}=183)$, eram insuficientemente ativas; com o avançar da idade, esses valores foram superiores, atingindo 69,1\% naquelas com mais de 70 anos. $\mathrm{Nahas}^{23}$ cita em seus estudos que pessoas que se mantêm inativas fisicamente (sedentárias) ou 
irregularmente ativas possuem maiores riscos relacionados à saúde interferindo na qualidade de vida. Matsudo et al. ${ }^{24}$ verificaram que a capacidade física de mulheres da sexta à oitava década de vida, participantes de um programa regular de atividade física, permaneceram estáveis.

No estudo realizado por Marin et al., ${ }^{25}$ com mulheres acima de 50 anos, na avaliação da força muscular, verificou-se que a perda foi maior em membros inferiores, quando comparados aos membros superiores. Esse fenômeno talvez possa ser explicado pela síndrome do desuso, quando ocorre diminuição na frequência das atividades que envolvem tais grupos musculares, pelo fato de os indivíduos passarem a realizar a maior parte das atividades na posição sentada. O mesmo não acontece com os membros superiores, que são bem mais requisitados para atividades de vida diária. Entretanto, pode-se analisar que a prática regular de exercícios físicos pode ser considerada o principal componente de melhora do nível de atividade física.

Em relação aos idosos considerados irregularmente ativos, os quais realizam atividades físicas insuficientes, realizando no máximo 30 minutos ou menos de atividade física por dia, verificou-se que, no G1, 24\% se enquadram neste perfil, enquanto no G2 foram totalizados $20 \%$. Matsudo ${ }^{26}$ mostra que a atividade física no tempo livre realizada em pouca quantidade, em intensidade leve duas vezes ou mais na semana e atividades de condicionamento realizadas menos de três vezes por semana foram associadas com dificuldades na mobilidade de adultos e idosos. Em outro estudo, Matsudo et al. ${ }^{27}$ constataram que o nível de atividade física de mulheres entre 50 e 69 anos e mais de 70 anos foram, respectivamente, irregularmente ativo $(32,1 \%)$ e sedentário $(34,6 \%)$. Estes achados vão de encontro ao presente estudo, que verificou maior número de idosos irregularmente ativos do que sedentários.

Com o intuito de realizar associação entre o nível de atividade física e a qualidade de vida em ambos os grupos, foi observada semelhança no percentual de indivíduos ativos em cada grupo (60\% para G1 e 56\% para G2); entretanto, os valores não foram os mesmos quando relacionados à qualidade de vida. $\mathrm{O}$ G1 apresentou escore maior $(10,64 \pm 7.79)$, afirmando que apresenta qualidade de vida inferior quando comparado ao G2 $(5,24 \pm 5.54)$. Por outro lado, o G1 obteve associação de $50 \%$ entre as duas variáveis, indicando menor interação entre o nível de atividade física e a qualidade de vida, o que pode ser explicado pela ausência de exercícios físicos.

Este estudo constatou que indivíduos considerados ativos e que praticam exercícios físicos têm uma qualidade de vida melhor, se comparados aos indivíduos ativos que não praticam exercícios físicos. Essa diferença se dá pelo fato de os praticantes de exercícios físicos conviverem mais tempo em grupo, sentiremse capacitados e independentes, ${ }^{28}$ facilitando a participação dos mesmos na sociedade e possibilitando a realização das atividades cotidianas. Assim, idosos que apenas executam as tarefas de vida diária, apesar de se manterem ativos, não apresentam acréscimos significativos em sua capacidade funcional.

Segundo Kuwano \& Silveira, ${ }^{29}$ idosos que não praticam atividade física preservam sua capacidade funcional pela história de vida e pelas atividades cotidianas que realizam. Entretanto, o processo de perda funcional com o envelhecimento é significativo e rápido, sendo que a atividade física deve ser recomendada para manter e minimizar as perdas funcionais - consequentemente, contribuindo para uma melhor qualidade de vida. De acordo com Borges \& Moreira, ${ }^{30}$ mesmo quando a prática regular de exercício físico for iniciada em uma fase tardia da vida, seus adeptos conquistam melhor qualidade de vida e recuperam ou mantêm sua autonomia funcional.

Dessa forma, espera-se que programas de treinamento físico promovam a socialização e o divertimento, contribuindo para a adesão e permanência dos participantes. De forma geral, Aguiar ${ }^{31}$ afirma que a prática de exercícios físicos na terceira idade deve ser estimulada, 
pois melhora a qualidade de vida relacionada ao domínio físico, à força e à flexibilidade, contribuindo para a prevenção de lesões e a independência do idoso.

\section{CONCLUSÕES}

Pode-se concluir que o nível de atividade física nem sempre está relacionado à prática de

\section{REFERÊNCIAS}

1. Herédia VBM, Ferla AA, Lorenzi DRSD.

Envelhecimento, saúde e políticas públicas. Caxias do Sul- RS: Educs, 2007.

2. IBGE. Indicadores sociodemográficos e de saúde no Brasil. Rio de Janeiro: IBGE, 2009

3. Wold Health Organization. Health of the elderly. Geneva: Wold Health Organization; 1989. WHO Technical Report Series 779.

4. Robergs RA, Roberts SO. Princípios fundamentais de fisiologia do exercício para aptidão, desempenho e saúde.1.ed. São Paulo:Phorte, 2002.

5. Reeves ND, Narici MV, Maganaris CN. Myotendinous plasticity to ageing and resistance exercise in humans. Exp. Physiol. 2006; 9(3): 483-498.

6. ACSM. Programa de condicionamento físico da ACSM. 2.ed. São Paulo: Manole, 1999.

7. Marotti J, Galhardo APM. Furuyama RJ, Pigozzo MN, Campos TN, Laganá DC. Amostragem em Pesquisa Clínica: tamanho da amostra. Rev. de Odont. da Univ. Cid. de São Paulo. 2008; 20(2): 186-194.

8. Benedetti TB, Mazo GZ, Barros MVG. Aplicação do questionário internacional de atividades físicas para avaliação do nível de atividades físicas de mulheres idosas: validade concorrente e reprodutibilidade teste-reteste. R. bras. Ci e Mov. 2004; 12(1): 25-34.

9. Matsudo SM, Matsudo VR, Andrade D, Araújo T, Oliveira L, Andrade E, et al. Nível de atividade física da população do Estado de São Paulo: análise de acordo com o gênero, idade, nível socioeconômico, distribuição geográfica e de conhecimento. Rev. Bras. Ciên. e Mov. 2002; 10 (4): 41-50.

10. Moura RMF,Lima RCM, Lage DC, Amaral EAA. Efeitos do treinamento aeróbio na qualidade de vida e na capacidade funcional de indivíduos hemiparéticos crônicos. ACTA FISIATR. 2005; 12(3): 94-99. exercícios físicos, pois o mesmo engloba outros fatores, como as atividades de vida diária.

Neste estudo, idosos sedentários apresentaram bons níveis de atividade física, entretanto os idosos que praticavam exercícios físicos obtiveram melhor qualidade de vida, sugerindo que o exercício físico pode ser fator determinante para um aumento nos níveis de atividade física e, consequentemente, na melhora da qualidade de vida do idoso.

11. Pauli JR, Souza LS, Zago AS, Gobbi S. Influência de 12 anos de prática de atividade física regular em programa supervisionado para idosos. Rev Bras Cineantropom Desempenho Hum. 2009; 11(3): 255-260.

12. Toscano JJO, Oliveira ACC. Qualidade de Vida em Idosos com Distintos Níveis de Atividade Física. Rev Bras Med Esporte. 2009; 15(3).

13. Nahas MV. Atividade física, Saúde e Qualidade de vida: conceitos e sugestões para um estilo de vida ativo. 2a ed. Londrina: Midiograf, 2001.

14. Ghorayeb N, Barros Neto TL. O exercício: preparação fisiológica, avaliação médica, aspectos especiais e preventivos. São Paulo: Atheneu, 1999.

15. Okuma SS. O idoso e a atividade física: Fundamentos e pesquisa. Campinas: Papirus, 1998.

16. American College of Sports Medicine Position Stand. Exercise and physical activity for older adults. Med. Sci. Sports Exerc. 1998; 30(6):992-1008.

17. Mazo GZ. Atividade física e qualidade de vida de mulheres idosas. Rev. Bras. de Ciên.do Enve. Hum. 2008: 115-118.

18. Cosme RG, Okuma SS, Mochizuki LA. Capacidade funcional de idosos fisicamente independentes praticantes de atividade física. Rev. bras. Ci. e Mov. 2008; 16(1): 39-46.

19. Hernandes ESC, Barros JF. Efeitos de um programa de atividades físicas e educacionais para idosos sobre o desempenho em testes de atividades da vida diária. Rev. bras. Ci. e Mov. 2004; 12(2): 43-50

20. Mazo GZ, et al. O exercício físico pode contribuir com mudanças positivas e aumentar a capacidade física do idoso. Nível de atividade física, condições de saúde e características sócio-demográficas de mulheres idosas brasileiras. Rev Port Cien Desp. 2003; 2(5) 202-212. 
21. Hallal PC. Victora CG, Wells JCK, Lima RC. Physical inactivity: prevalence and associated variables in Brazilian adults. Med. and Sci. in Spo. and Exer. 2003; 35(11):1894-1900.

22. Brach JS, et al. The Association between physical function and lifestyle activity and exercise in the health, aging and body composition study. J. Am. Geriatr Soc. 2004; 52: 502-509.

23. Nahas MV. Atividade física, Saúde e Qualidade de vida: conceitos e sugestões para um estilo de vida ativo. 2a ed. Londrina: Midiograf, 2001.

24. Matsudo SM, et al. Nível de atividade física da população do Estado de São Paulo: análise de acordo com o gênero, idade, nível socioeconômico, distribuição geográfica e de conhecimento. Rev. Bras. Ciên. e Mov. 2002; 10 (4): 41-50.

25. Marin RV, et al. Acréscimo de $1 \mathrm{~kg}$ aos exercícios praticados por mulheres acima de 50 anos: impacto na aptidão física e capacidade funcional. Rev. Bras. Ciên. e Mov. 2003; 11(1): 53-58.
26. Matsudo SM. Atividade física na promoção da saúde e qualidade de vida no envelhecimento. Rev. bras. Educ. Fís. Esp. 2006; 20(5): 135-37.

27. Matsudo SM, et al. Evolução do perfil neuromotor e capacidade funcional de mulheres fisicamente ativas de acordo com a idade cronológica. Rev Bras Med Esporte 2003; 9(6): 365-376

28. Aragão JCB, Dantas E, Dantas B. RML e Autonomia. Rev. Fit. e Per. Jour. 2002; 1(3): 29-38.

29. Kuwano VG; Silveira AM. A influência da atividade física sistematizada na autopercepção do idoso em relação às atividades da vida diária. R. da Educação Física/UEM, 2002; 13(2): 35-39.

30. Borges MRD, Moreira AK. Influências da prática de atividades físicas na terceira idade: estudo comparativo dos níveis de autonomia para o desempenho nas AVDs e AIVDs entre idosos ativos fisicamente e idosos sedentários. Motriz. 2009; 15(3): 562-573.

31. Aguiar JB, Gurgel LA. Investigação dos efeitos da hidroginástica sobre a qualidade de vida, a força de membros inferiores e a flexibilidade de idosas. Rev. bras. Educ. Fís. Esporte. 2009; 23(4): 335-44. 